\title{
Pré-natal odontológico: noções de interesse
}

Dental prenatal: notions of interest

Prenatal dental: nociones de interese

\author{
Marília Rodrigues Moreira ${ }^{1}$; Gabriela Cristina Santin ${ }^{2}$; Leonardo Gontijo Matos ${ }^{3}$; Danuze Batista \\ Lamas Gravina ${ }^{4}$;
}

\section{Resumo}

Apesar dos avanços na Odontologia, muitos cirurgiões dentistas ainda possuem a concepção de que o tratamento odontológico à gestante deve ser postergado e que procedimentos como tomadas radiográficas e anestesias não devem ser realizados. Entretanto sabe-se que nesse período a gestante constitui um grupo de risco uma vez que as mudanças hormonais, físicas e psicológicas podem desencadear um desequilíbrio à saúde bucal da mulher. Dessa forma, o objetivo desta revisão de literatura foi esclarecer e atualizar as recomendações sobre o pré-natal odontológico. A literatura científica mostra que o atendimento odontológico as gestantes é seguro e que apresenta muitos benefícios, tanto para a mãe quanto para o próprio bebê.

Palavras-chave: Gestantes, Saúde Bucal, Protocolos Clínicos.

\section{Abstract}

Despite the advances in dentistry, many dentists still have the idea that dental treatment to pregnant women should be delayed and that procedures such as X-ray and anesthesia taken should not be performed. However it is known that during this period the mother is a risk group as the hormonal, physical and psychological changes can trigger an imbalance to the oral health of the woman. Thus, the purpose of this review was to clarify and update the recommendations on dental prenatal care. The scientific literature shows that dental care in pregnant women is safe and has many benefits for both the mother and the baby itself.

Key-words: Pregnant Women, Oral Health, Clinical Protocols.

\section{Resumen}

A pesar de los avances en la odontología, muchos dentistas todavía tienen la idea de que el tratamiento dental a mujeres embarazadas debe retrasarse y que los procedimientos como los rayos $\mathrm{X}$ y la anestesia tomada no deben realizarse. Sin embargo, se sabe que en este período, la madre es un grupo de riesgo por los cambios hormonales, físicos y psicológicos que pueden provocar un desequilibrio en la salud bucal de la mujer. Por lo tanto, el objetivo de esta revisión es aclarar y actualizar las recomendaciones sobre el cuidado prenatal dental. La literatura científica muestra que el cuidado dental en las mujeres embarazadas es seguro y tiene muchos beneficios tanto para la

\footnotetext{
${ }^{1}$ Doutora em Odontopediatria pela Faculdade de Odontologia de Ribeirão Preto da Universidade de São Paulo (FORPUSP). Professora e coordenadora dos cursos de especialização em Odontopediatria da Associação Brasileira de Odontologia, Seção Uberlândia e Promove-Uberaba. Avenida Brasil, n 4592, Bairro Umuarama, Uberlândia-MG, Brasil, CEP: 38405-312. e-mail: marilia.moreira@ig.com.br

${ }^{2}$ Doutora em Odontopediatria pela Faculdade de Odontologia de Ribeirão Preto da Universidade de São Paulo (FORPUSP). Professora adjunta do Departamento de Odontologia da Universidade Estadual de Maringá. Rua Dr. Alberto Byington Jr., n 201, Vila Esperança, Maringá-PR, Brasil. CEP: 87020-380. e-mail: gabsantin1310@ gmail.com

${ }_{3}^{3}$ Mestre em Ortodontia pela Pontifícia Universidade Católica de Minas Gerais. Rua Tiradentes, n.260, Bairro Centro, Patos de Minas-MG, Brasil, CEP: 38700-134. e-mail: leonardo.gontijo@terra.com.br

${ }^{4}$ Mestre em Ciências da Saúde pela Universidade de Brasília. Rua SQSW, n. 305, Sudoeste, Brasília-DF, Brasil. email: danuzeblamas@globo.com
} 
ISSN 2179-6750

madre como para el bebé en sí.

Palabras-claves: Mujer Embarazada, Salud Oral, Protocolos Clínicos.

\section{Introdução}

Na Odontologia, a abordagem à gestante tornou-se uma realidade incontestável, despertando notável interesse no âmbito da profissão. A gestação é um período especial na vida da mulher e caracteriza-se por uma série de alterações sistêmicas e com repercussões na cavidade bucal, como hipersecreção das glândulas salivares e tendência a náuseas e vômitos ${ }^{1}$. Além disso, há um aumento da vascularização do periodonto que, quando associada à deficiência no controle do biofilme dental, pode acarretar complicações, como o parto prematuro ${ }^{1,2}$.

Por ser um período de mudanças fisiológicas e psicológicas complexas, cada gestante vivencia de forma distinta estas transformações que podem gerar medos, dúvidas, angústias e/ou curiosidades em saber o que acontece no interior do seu corpo e com seu bebê. Dessa forma, muitas gestantes procuram orientações que assegurem o bem-estar do seu bebê, sendo este período uma boa oportunidade para a realização de atividades de educação e promoção de saúde, sendo esse o principal objetivo do pré-natal odontológico ${ }^{3}$.

Entretanto, apesar de sua importância, o pré-natal odontológico ainda é negligenciado pelas gestantes, tanto pela falta de conhecimento da real importância deste acompanhamento ${ }^{4}$, quanto pelo medo de se submeter a certos procedimentos, como as tomadas radiográficas ${ }^{5,6}$. Assim, cabe ao cirurgião dentista o conhecimento e segurança em orientar e atender essa população. Dessa forma, o objetivo desta revisão de literatura é abordar os principais problemas odontológicos e o atendimento durante a gestação.

\section{Problemas odontológicos de interesse na gestação}

\section{Alterações periodontais}

Uma pesquisa realizada por Offenbacher et al. ${ }^{7}$ (1996), demonstrou que infecções maternas durante a gravidez podem estimular a ocitocina (hormônio que promove contrações uterinas), levando a ocorrência de parto prematuro e o baixo peso do bebê ao nascer. Além disso, verificou que o aumento do nível de Prostaglandina $\mathrm{E}_{2}\left(\mathrm{PGE}_{2}\right)$ no fluído crevicular pode ser considerado um marcador de atividade da doença periodontal, estando também associado com o baixo de peso do recém-nascido.

Os efeitos da gravidez sobre a inflamação gengival são sentidos a partir do segundo mês de gestação, podendo causar a chamada gengivite gravídica. A exacerbação da resposta inflamatória 
ISSN 2179-6750

estaria associada à elevação dos níveis plasmáticos dos hormônios estrogênio e progesterona ${ }^{8,9} \mathrm{e} a$ ligação destes a receptores específicos, aumentando a permeabilidade vascular e edema dos tecidos gengivais $^{10,110,12}$. Outro fator possivelmente associado, seria as alterações do sistema imunológico materno, ocasionando redução da resposta dos tecidos gengivais contra fatores irritantes locais gengivais ${ }^{10,11,12}$. Ainda associado a fatores hormonais, um possível desequilíbrio desses hormônios parece ser capaz de influenciar a microbiota normal e induzir uma alteração significante na proporção de bactérias anaeróbias/aeróbias da região subgengival ${ }^{13}$.

Porém, tanto a presença de biofilme dental quanto a atuação hormonal, isoladamente, não podem ser considerados fatores para o desenvolvimento da gengivite durante a gravidez, sendo necessária a presença de ambos, assim como a de receptores hormonais e a especificidade da resposta imune do hospedeiro ${ }^{8,9}$. Apesar desses fatores, a gengivite gravídica pode ser prevenida e desaparecer alguns meses após o parto desde que os irritantes locais sejam controlados, como a remoção do biofilme dental por meio de higiene bucal adequada e/ou profilaxia profissional $14,15,16,17$

Além da gengivite gravídica, outro achado, porém não tão comum, é o tumor gravídico, uma lesão benigna que surge geralmente no primeiro trimestre da gestação, resultante de agressões repetitivas, micro-traumatismo e irritação local sobre a mucosa gengival. Apresenta características semelhantes ao granuloma piogênico e ocorre preferencialmente na região anterior da maxila, por vestibular. A remoção cirúrgica é indicada nos casos em que houver interferência na mastigação, na execução da higiene bucal ou em situações de ulceração; caso contrário os irritantes locais devem ser removidos e o tumor proservado até o pós-parto, quando normalmente ocorre sua redução espontânea $^{18}$.

\section{Cárie dentária}

Com relação à alteração microbiológica, além da variação hormonal, algumas mulheres podem ainda apresentar mudanças de hábitos como a "Síndrome da perversão do apetite", a qual determina um aumento da frequência alimentar e um acréscimo do apetite por alimentos açucarados, o que acarreta o aumento do nível de bactérias cariogênicas ${ }^{1}$.

Apesar da gravidez não ser responsável pelo aparecimento de lesões cariosas e pela perda de minerais dos dentes da mãe, o aumento da atividade cariogênica pode estar relacionado com a alteração da dieta e com a presença de biofilme dental ${ }^{19,20}$. Além disso, em alguns casos a gestante pode negligenciar a higiene bucal e, devido a maior exposição do esmalte ao ácido gástrico (vômitos); alterações de hábitos alimentares resultantes do fato de estar grávida; aumento da 
ISSN 2179-6750

frequência das refeições (com a compressão do feto, diminui a capacidade volumétrica do estômago e, consequentemente, a gestante alimenta-se em pequenas quantidades, porém mais vezes), aumenta-se a probabilidade do desenvolvimento da cárie dentária ${ }^{19,20}$.

É importante ressaltar que o fluxo salivar e a composição da saliva podem ser alterados durante a gestação em decorrência da variação hormonal aumenta nos primeiros meses da gestação e a hiperatividade das glândulas salivares é um fenômeno, sem causa definida. O excesso de secreção salivar provoca náusea e vômito e, se persistir até o final da gestação, provoca queda da capacidade tampão da saliva - fator importante no aumento do risco de desmineralização dental ${ }^{21}$.

Sobre a prevenção da cárie dentária no bebê, essa pode ser desenvolvida desde a vida intrauterina, pois a partir do quarto mês de gestação inicia-se a formação do paladar do bebê; portanto, a implementação de novos hábitos alimentares da mãe proporcionará uma melhor escolha na dieta pela criança ${ }^{20}$.

\section{Atendimento odontológico na gestação}

A gestação traz consigo um preconceito enorme em relação ao tratamento odontológico. $\mathrm{O}$ mito de que "seus dentes irão estragar e sua gengiva sangrar", assim como, os relatos de que medicamentos podem ser deletérios para a saúde do bebê estão incutidos de maneira muito forte na nossa sociedade. A causa dessa desinformação pode ser atribuída aos profissionais de saúde que, por muito tempo, acreditaram nessas ideias e não tiveram a preocupação de encaminhar as gestantes para uma avaliação odontológica ${ }^{22}$.

Vários são os aspectos que devem ser observados no atendimento odontológico da paciente gestante. Inicialmente é necessário esclarecer que ela não só pode como deve passar por acompanhamento odontológico a fim de minimizar ou evitar os problemas associados, como os relatados anteriormente.

Apesar de a prevenção odontológica ser priorizada, em casos onde há a necessidade de intervenção, o tratamento deve ser instituído, uma vez que os problemas da cavidade bucal podem ter influência tanto para a mãe quanto para o feto, especialmente quando se compromete a nutrição e contribui-se para a infecção e disseminação de patógenos no sangue ${ }^{23}$. O período ideal e mais seguro para o tratamento odontológico é durante o segundo trimestre da gestação. No entanto, os casos que necessitam tratamento de urgência devem ser solucionados sempre, independentemente do período gestacional. A maioria dos procedimentos odontológicos pode ser realizada durante a gravidez, observando-se alguns cuidados como: planejar sessões curtas; adequar a posição da cadeira e evitar consultas matinais, já que neste período as gestantes têm mais ânsia de vômito e 
ISSN 2179-6750

risco de hipoglicemia. Exodontias não complexas, tratamentos periodontal e endodôntico, restaurações dentárias, instalação de próteses e outros tipos de procedimentos devem ser realizados com segurança, de preferência no segundo trimestre da gestação. Tratamentos seletivos como reabilitações bucais extensas e cirurgias mais invasivas podem ser programadas para o período de pós-parto $^{24}$.

\section{Uso de medicamentos}

É de extrema importância avaliar os possíveis riscos e benefícios da indicação de qualquer substância terapêutica na gravidez, principalmente durante os três primeiros meses $\left(18^{\circ}\right.$ ao $60^{\circ}$ dia $)$ de gestação, pois neste período ocorre a organogênese, fase em que os órgãos do feto estão em desenvolvimento, sendo considerado um período crítico para a suscetibilidade teratogênica. Já no período fetal $\left(60^{\circ}\right.$ dia até o final da gestação), período de crescimento e aperfeiçoamento das funções, alguns medicamentos também podem determinar alterações funcionais em certos órgãos ${ }^{25}$.

Atualmente, o analgésico mais amplamente prescrito pelo cirurgião-dentista é sem dúvida o paracetamol (Dôrico®, Tylenol®), podendo ser prescrito também a pacientes gestantes e lactantes 26. A dipirona sódica (Novalgina ${ }^{\circledR)}$ é o analgésico de segunda escolha, pois pode provocar agranulocitose, redução do número de granulócitos no sangue periférico (neutropenia), podendo predispor o indivíduo às infecções.

Os anti-inflamatórios não-esteroidais (AINES) são representados por um grande número de fármacos, dentre estes, o ácido acetilsalicílico, diclofenaco, ibuprofeno, naproxeno, indometacina, rofecoxib, que são prescritos com frequência pelo cirurgião-dentista. O ácido acetilsalicílico (Aspirina $\left.{ }^{\circledR}\right)$ deve ser usado com cautela, pois além do risco de prolongar o trabalho de parto, ao inibir síntese das prostaglandinas envolvidas na iniciação das contrações uterinas, há também algumas evidências de que, em doses muito elevadas, pode causar efeitos teratogênicos ${ }^{26}$.

Os corticosteróides devem ser usados com muita cautela, pois em doses elevadas pode causar anormalidade na curva glicêmica da gestante, insuficiência das supra-renais e a síndrome de Cushing, também chamada de hipercortisolismo ou hiperadrenocorticismo, sendo essa uma desordem endócrina que pode ocorrer devido aos níveis elevados de cortisol no sangue induzidos pela administração do medicamento ${ }^{26}$.

Quanto aos antibióticos, o metronidazol (Flagyl®) não deve ser administrado durante a gestação e lactação, pois é tido como potencialmente teratogênico. Em casos de real necessidade, pode ser utilizada a amoxicilina (Amoxil@), benzilpenicilina, benzatina (Benzetacil@), eritromicina $\left(\right.$ Pantomicina $\left.{ }^{\circledR}\right)$, fenoximetilpenicilina potássica $\left(\text { Pen-Ve-Oral }{ }^{\circledR}\right)^{26}$. 
ISSN 2179-6750

O uso de ansiolíticos como o bromazepam (Lexotan®), lorazepam (Lorax®), diazepam (Dienpax $\left.{ }^{\circledR}\right)$, Valium $\left.{ }^{\circledR}\right)$ estão contra-indicados durante a gravidez, pois se suspeita que estes medicamentos tenham um poder teratogênico razoável ${ }^{20,26,27}$.

Em relação ao uso de anestésicos, a solução anestésica de escolha é a lidocaína $2 \%$ com epinefrina (1:100.000) e um limite máximo de dois tubetes $(3,6 \mathrm{ml})$ por sessão, procedendo sempre injeção lenta da solução ${ }^{28}$.

\section{Exame Radiográfico}

O exame radiográfico, como um exame complementar, pode ser realizado se tomada todas as medidas de precaução (uso de filmes ultrarrápido e avental de chumbo), levando em consideração que a dose recebida em uma radiografia dentária é muito inferior aquela que pode causar más formações congênitas ${ }^{29}$.

\section{Uso dos fluoretos}

Outro ponto bastante questionado na Odontologia é sobre a prescrição de fluoretos em gestantes. O conhecimento atual sobre o mecanismo de ação do fluoreto indica que seu efeito é predominantemente tópico, ocorrendo principalmente na interface biofilme/esmalte dental, através da remineralização de lesões de cárie iniciais e da redução da solubilidade do esmalte dentário. Portanto, a prescrição de medicamentos fluoretados no período pré-natal não traz beneficio que justifique sua indicação ${ }^{29,30,31}$.

\section{Conclusão}

A gestação é um estado singular e valioso no ciclo de vida da mulher. As gestantes constituem temporariamente pacientes especiais do ponto de vista odontológico devido às mudanças psicológicas, físicas e hormonais, criando condições adversas ao meio bucal ${ }^{1,22}$. O atendimento odontológico é um assunto bastante controverso, principalmente em função dos mitos que são baseados em crenças antigas sem fundamentação científica, tanto por parte da gestante quanto por parte dos cirurgiões-dentistas que não se sentem seguros em atendê-las ${ }^{27}$.

O conhecimento dos cirurgiões-dentistas sobre os trimestres na gravidez é importante para mensurar e poder prever possíveis problemas, possibilitando cuidados odontológicos na prescrição de medicamentos e exames radiográficos, induzindo a um tratamento seguro, eficaz e com menor risco de efeitos deletérios aos bebês, segundo os investigadores ${ }^{27}$.

Do ponto de vista da formação do feto, o atendimento odontológico às gestantes deve ser 82 
ISSN 2179-6750

preferencialmente realizado no segundo trimestre de gestação, mas em casos de urgência, qualquer época é aceitável, já que nenhuma necessidade deve ser negligenciada pelo medo de colocar em risco a saúde do bebê, tendo em vista que a presença de infecção e inflamação na mãe pode acarretar problemas de alta severidade ${ }^{23,27}$.

Entretanto, a realidade que predomina na assistência odontológica à população em geral é que quase sempre o cirurgião-dentista se omite em sua função de facilitador da aprendizagem em saúde bucal. O fato da maioria das gestantes estarem ainda desinformadas sobre o assunto deveria ser motivo de preocupação, pois são as mães as principais fontes de transmissão de microorganismos patogênicos e as principais responsáveis pela educação no núcleo familiar ${ }^{29}$.

A assistência ao pré-natal de qualidade é essencial para redução dos elevados índices de mortalidade materna e peri-natal verificados no Brasil. Essa atenção visa ainda trabalhar com as gestantes a educação em saúde na tentativa de sensibilizá-las e motivá-las para melhor cuidarem da sua saúde e também da saúde de seus filhos ${ }^{30,31}$.

\section{Referências}

1. Granville-Garcia AF, Leite AF, Smith LEA, Campos RVS, Menezes VA. Pregnant women's knowledge of oral health in the city of Caruaru - PE. Rev Odontol UNESP, 2007; 36(3):2439.

2. Bragion DB, Costa SRG, Zaffalon GT, Tognetti VM, Garcia MBO. Periodontal diseases and premature obstetric labor. Is there a relationship of risk? Braz J Health, 2012;3(2):1-10.

3. Konish F, Konish R. Odontologia intrauterina: um novo modelo de construção de saúde bucal. In: Cardoso AJR, editor. Odontopediatria: Prevenção. São Paulo: Artes Médicas; 2002. p. 155-65.

4. Massoni ACLT, Pereira RB, Nóbrega DRM, Costa LED, Fernandes JMFA, Rosenblatt A. Avaliação do conhecimento de gestantes e puérperas primíparas e multíparas sobre cárie dentária. RGO, Rev. Gaúch. Odontol. [periódico na Internet]. 2015 [acessado em 2015 Nov 25]; 63(2):145-52. Disponível em: http://www.scielo.br/scielo. php?script=sci_arttext\&pid=S1981-86372015000200145\&lng=en\&nrm=iso.

5. Santos Neto ET, Oliveira AE, Zandonade E, Leal MC. Acesso à assistência odontológica no acompanhamento pré-natal. Ciênc Saúde Colet 2012; 17(11):3057-68.

6. Nogueira LT, Valsecki Júnior A, Martins CR, Rosell FL, Silva SRC. Retardo na procura do tratamento odontológico e percepção da saúde bucal em mulheres grávidas. Odontol. Clín.Cient. (Online) [periódico da Internet]. 2012 [acessado em 2015 Nov 25]; 11(2):127-31. 
ISSN 2179-6750

Disponível em: http://revodonto.bvsalud.org/scielo.php?script=sci_arttext \&pid=S1677$38882012000200008 \& \operatorname{lng}=$ pt\&nrm=iso

7. Offenbacher S, Katz V, Fertik G, Collins J, Boyd D, Maynor G, et al. Periodontal infection as a possible risk factor for preterm low birth weight. J Periodontol, 1996; 67:1103-13.

8. Tunes UR, Rapp GE. Influência das condições sistêmicas sobre as doenças periodontais. Atualização em Periodontia e Implantodontia. São Paulo: Artes médicas, 1999.

9. Yalcin F, Basegmez C, Isik G, Berber L, Eskinazi E, Soydinc M, et al. The effects of periodontal therapy on intracrevicular prostaglandin $E_{2}$ concentrations and clinical parameters in pregnancy. J Periodontol, 2002; 73(2):173-7.

10. Lapp AC, Thomas ME, Lewis JB. Modulation by progesterone of interleuykin-6 production by gingival fibroblasts. J Periodontol, 1999; 66(2):279-84.

11. Scavuzzi AIF, Rocha MCBS, Viana MIPl. Estudo da prevalência de doença periodontal em gestantes brasileiras residentes em Salvador - BA. Rev Odontol Brasil Central, 1999; $8(25): 40-5$.

12. Bastiani C, Cota ALS, Provenzano MGA, Fracasso MLC, Honório HM, Rios D. Conhecimento das gestantes sobre alterações bucais e tratamento odontológico durante a gravidez. Odontol Clín Cient [periódico na Internet]. 2010 [acessado 2015 Nov 25]; 1(1):16. Disponível em: http://revodonto.bvsalud.org/scielo.php?script=sci_arttext \&pid=S1677$38882010000200013 \& \operatorname{lng}=\mathrm{p} \& n r m=$ iso

13. Rodrigues AS, Lima DBGO, Ganhito JA, Romito GA, Lotufo RFG, Micheli G, et al. Parto prematuro e baixo peso ao nascer associados à doença periodontal: aspectos clínicos, microbiológicos e imunológicos. Rev Odontol UNICID, 2004; 16(1):55-61.

14. Jeffcoat MK, Hauth JC, Geurs NC, Reddy MS, Cliver SP, Hodgkins PM, et al. Periodontal disease and preterm birth: results of a pilot intervention study. J Periodontol, 2003; 74(2):1214-8.

15. Lopes AM, Bosco JMD, Gasparini LL, Kina JR. Fatores etiológicos associados com a gengivite na gravidez. Rev Paul Odontol, 2004; 26(4):31-4.

16. López NJ, Da Silva I, Ipinza J, Gutiérrez J. Periodontal therapy reduces the rate of preterm low birth weight in women with pregnancy associated gingivitis. J Periodontol, 2005; 76(1):2144-53.

17. Macones GA, Parry S, Nelson DB, Strauss JF, Ludmir J, Cohen AW, et al. Treatment of localized periodontal disease in pregnancy does not reduce the occurrence of preterm birth: 
ISSN 2179-6750

results from the Periodontal Infections and Prematurity Study (PIPS). Am J Obstet Gynecol, 2010; 202(2):147.e1-8.

18. Elias R. Atendimento estomatológico em gestantes: verdades e mitos. Rev ABO Nac, 2004; 11(6):333-4.

19. Peres SHCS, Cardoso MTV, Garcez RMVB, Peres AS, Bastos JRM. et al. Tratamento alternativo de controle da cárie dentária no período materno-infantil. Rev APCD, 2001; 55(5):346-51.

20. Reis DM, Pitta DR, Ferreira HMB, Jesus MCP, Moraes MEL, Soares MG. Educação em saúde como estratégia de promoção de saúde bucal em gestantes. Ciênc. Saúde Coletiva, 2010; 15(1):269-76.

21. Karnik AA, Pagare SS, Krishnamurthy V, Vahanwala SP, Waghmare M. Determination of salivary flow rate, $\mathrm{pH}$, and dental caries during pregnancy: A study. J Indian Acad Oral Med Radiol,, 2015;27:372-6.

22. Imparato PCJ, Echeverria SA. Gestante no Contexto do Atendimento Odontológico. J Bras Odontopediatr Odontol Bebê, 2002; 23(5):63-71.

23. Ramires I et al. Métodos de uso sistêmico dos fluoretos no controle da cárie dentária. In: Buzalaf MAR. Fluoretos e saúde bucal. São Paulo: Editora Santos; 2008. p.163-194.

24. Trevisan CL, Pinto AAM. Fatores que interferem no acesso e na adesão das gestantes ao tratamento odontológico. Arch Health Investigation, 2013; 2(2):1-9.

25. Carmo TA, Nitrini SAOO. Prescrições de medicamentos para gestantes: um estudo fármaco epidemiológico. Cad Saúde Pública, 2004; 20(4):1004-13.

26. Yagiela JA. Anestésicos locais. In: Yagiela JA, Neidle EA, Dowd FJ. Farmacologia e terapêutica para dentistas. $4^{\mathrm{a}}$ ed. Guanabara Koogan: Rio de Janeiro; 2000.

27. Vasconcelos RG, Vasconcelos MG, Alves Júnior RPMLC, Queiroz LMG, Barboza CAG. Atendimento odontológico a pacientes gestantes: como proceder com segurança. Rev Bras Odontol, 2012; 69(1):120-4.

28. Andrade ED. Terapêutica medicamentosa em odontologia. São Paulo: Artes Médicas; 2006.

29. Guimarães AO, Costa ICC, Oliveira ALS. As origens, objetivos e razões de ser da Odontologia para bebês. J Bras Odontopediatr Odontol Bebê, 2003; 29(6):83-6.

30. Alves CS, Bezerra MM. Atenção odontológica no pré-natal: a percepção das gestantes do Bairro Padre Palhano, Sobral-CE. Sanare 2005;6(1):61-8.

31. Codato LAB, Nakama L, Cordoni Júnior L, Higasi MSL. Atenção odontológica à gestante: papel dos profissionais de saúde. Ciênc. Saúde Coletiva, 2011; 16(4):2297-301. 